\title{
ISÓTOPOS DE OXIGÊNIO E TEMPERATURA DE FORMAÇÃO DOS VEIOS MINERALIZADOS COM WOLFRAMITA DA JAZIDA PEDRA PRETA, SUL DO PARÁ
}

\author{
FRANCISCO JAVIER RIOS*, RAIMUNDO NETUNO VILLAS**, KAZUO FUZIKAWA*, \\ ALCIDES NỎBREGA SIAL *** \& GORKI MARIANO****
}

\begin{abstract}
OXIGEN ISOTOPES AND FORMA TION TEMPERA TURE OF QUARTZ- WOLFRAMITE VEINS OF THE PEDRA PRETA DEPOSIT, PARA STATE, BRAZIL The Pedra Preta wolframite ore deposit contains the main known tungsten reserves of the Amazon. The wolframite occurs in a vein network that cuts both the Musa granite $(1.88 \mathrm{Ga})$ and the Andorinhas greenstone belt host rocks $(2.9 \mathrm{Ga})$. Oxygen isotopic studies were carried out on quartz of the Musa granite as well as on quartz, wolframite and white mica of the post-intrusive hydrothermal veins (PHV). In the lower levels of the ore deposit (at depths below $160 \mathrm{~m}$ from the present-day erosion surface) similar isotopic values $\left(\delta^{18} \mathrm{O}_{\text {uuart }}\right.$ $=7-8 \%$ have been found for quartz regardless of its mode of occurrence. The calculated $\delta^{18} \mathrm{O}_{\text {fluid }}$ in equilibrium with quartz fell in the range of -3.16 to $-0.10 \%$ from 240 to $300^{\circ} \mathrm{C}$, suggesting that O-depleted fluids had an important role in the PHV formation. These fluids correspond to those of aqueous-carbonic nature that were convectively driven to the Pedra Preta system and might have acquired a lighter oxygen isotope composition due to their long residence time in the surrounding metamorphic rocks. The PHV quartz from the upper levels show $\delta{ }^{18} \mathrm{O}$ in the $9.04-9.67 \%$ range with the corresponding equilibrium $\delta{ }^{18} \mathrm{O}_{\text {fluid }}$ varying from 1.53 to $-1.79 \%$ in that same thermal range. The $\delta{ }^{18} \mathrm{O}$ increase in both quartz and fluid is believed to have resulted from the $\mathrm{CO}_{2}$-enrichment of the ascending fluids that had been previously oxidized at the granite apical zones. Wolframite and white mica (phengite) also sampled in the PHV presented $\delta^{18} \mathrm{O}$ values of 0.85 and $5.44 \%$ respectively. Oxygen isotope geothermometry of quartz-wolframite yielded temperatures of $321{ }^{\circ} \mathrm{C}$ (Shieh \& Zhang 1991) and $356^{\circ} \mathrm{C}$ (Zhang et al. 1994) which are in fairly good agreement with the higher values determined by fluid inclusion microthermometry $\left(230-350^{\circ} \mathrm{C}\right)$. Using the quartz-muscovite pair (Shieh \& Zhang 1991), temperatures in the order of $410^{\circ} \mathrm{C}$ were obtained, but they seem to be unrealistic since the white mica (phengite) was one of the latest minerals to precipitate in the PHV.

Keywords: Musa granite, wolframite ore, oxygen isotopes, geothermometry
\end{abstract}

RESUMO A jazida de wolframita de Pedra Preta constitui a principal reserva de tungstênio conhecida na Amazônia. O minério ocorre num campo filoneano que corta tanto o granito Musa (1,88Ga) como as rochas do greenstone belt de Andorinhas (2,9Ga). Foram desenvolvidos estudos de isótopos de oxigênio no quartzo do corpo granítico bem como na wolframita, mica branca e quartzo dos veios hidrotermais principais (VHP) que se alojaram após o posicionamento da intrusão. Observou-se uma similaridade entre os valores isotópicos do quartzo do granito e dos VHP $\left(\delta^{18} \mathrm{O}_{\text {quartzo }}=7-8 \%\right.$ oo no setor inferior da jazida (a profundidades abaixo de $160 \mathrm{~m}$ a partir da atual superfície de erosão), o que deve refletir equilíbrio com um mesmo fluido. Os valores de $8^{18} \mathrm{O}_{\mathrm{f}}$ ido no intervalo termal de 240 a $300^{\circ} \mathrm{C}$ foram calculados entre $-3,16$ e $-0,10^{\circ} \%$, sugerindo a participação de fluidos empobrecidos em ${ }^{18} \mathrm{O}$ na formação dos VHP. Esses fluidos são provavelmente os de natureza aquo-carbônica e caráter redutor (presença de $\mathrm{CH}_{4}$ ) que foram identificados nos estudos de inclusões fluidas e que entraram no sistema hidrotermal Pedra Preta após longo tempo de residência nas rochas das circunvizinhanças. Já o quartzo do VHP do setor superior da jazida mostrou valores de $\delta^{18} \mathrm{O}$ na faixa de 9,04 a $9,67 \%$ com valores de equilíbrio de $\delta^{18} \mathrm{O}_{\text {flúdo }}$ na faixa de $-1,79 / 1,53 \%$ o calculados para aauele mesmo intervalo termal. Credita-se ao $\mathrm{CO} 2$ produzido pela oxidação daqueles fluidos nas zonas apicais do granito o enriquecimento em ${ }^{18} \mathrm{O}$ tanto do quartzo como dos fluidos presentes no setor superior da jazida. A wolframita e a mica branca amostradas nos veios VHP apresentaram valores de $\delta^{18} \mathrm{O}$ de 0,85 e $5,44 \%$ respectivamente. $\mathrm{O}$ geotermômetro com base nos isótopos de oxigênio do par quartzo-wolframita forneceu temperaturas de $321^{\circ} \mathrm{C}$ (Shieh \& Zhang 1991 ) e $356^{\circ} \mathrm{C}$ (Zhang et al. 1994) que são plenamente compatíveis com os mais altos valores obtidos com os dados microtermométricos em inclusões fluidas $\left(230-350^{\circ} \mathrm{C}\right)$. Usando o par quartzo-muscovita (Shieh \& Zhang 1991), foram obtidas temperaturas de cerca de $410^{\circ} \mathrm{C}$ que parecem muito altas para a precipitação da mica (fengita), a qual foi um dos últimos minerais a se formar nos VHP.

Palavras-chave: Granito Musa, jazida de wolframita, isótopos de oxigênio, geotermometria.

INTRODUÇÃO A jazida de wolframita de Pedra Preta, localizada próximo à borda oeste do granito Musa $(1,88 \mathrm{Ga}$; Machado et al. 1991), na região de Rio Maria, sul da Província Mineral de Carajás, constitui a principal reserva de tungstênio conhecida na Amazônia. Foram calculadas 508.300 toneladas de minério, com teor médio de $1,01 \%$ de $\mathrm{WO}_{3}$ (Cordeiro et al. 1988).

Do ponto de vista metalogenético, trata-se de um depósito hidrotermal em cuja formação foram importantes tanto fluidos de derivação magmática como aqueles provenientes das rochas encaixantes e que convergiram para as zonas fraturadas da área de influência da intrusão granítica (Rios 1995). Dados mineralógicos e geoquímicos revelam marcantes diferenças entre os setores superior e inferior da jazida, que têm como linha divisória a profundidade de aproximadamente $160 \mathrm{~m}$ a partir da atual superfície de erosão.

Estudos isotópicos de oxigênio em minerais do corpo granítico e dos veios hidrotermais ressaltaram ainda mais essas diferenças e também serviram para fins geotermométricos, dando maior consistência ao modelo metalogenético que havia sido proposto (Rios 1995). A apresentação e discussão desses dados isotópicos justificaram a presente comunicação, mormente quando se constata a carência de estudos de isótopos estáveis em depósitos minerais brasileiros e se reconhece sua grande utilidade na elucidação da origem e evolução dos fluidos a partir dos quais os minérios foram precipitados.

GEOLOGIA DA JAZIDA A mineralização de wolframita ocorre num campo filoneano que corta, em profundidade, a cupola do granito Musa bem como as rochas do greenstone belt do Supergrupo Andorinhas (Docegeo 1988) que o hospeda. Essa unidade é composta pelos grupos Babaçu, dominado por rochas metavulcânicas básicas, e Lagoa Seca, dominado por rochas metassedimentares e datado em
2,9Ga (Macambira \& Lancelot 1992, Pimentel \& Machado 1994). O granito Musa faz parte da suíte intrusiva anorogênica paleoproterozóica que se instalou no bloco leste do Cráton Amazônico por volta de 1,9 Ga (Dall'Agnol et al. 1994). Nesse corpo foram mapeados monzo e sienogranitos como as principais fácies petrográficas, ambas compostas de quartzo, feldspato potássico, oligoclásio, biotita \pm hornblenda (Gastai 1987). Na jazida, desvendado por furos de sondagem, ocorre um biotita-microgranito de tonalidade rósea e textura heterogranular fina com evidências de greisenização.

Trabalhos anteriores ocuparam-se com os aspectos geológicos, petroquímicos e genéticos (Cordeiro et al. 1984, Gastai 1987, Santos 1987). Novos estudos metalogenéticos realizados na jazida (Rios 1995) permitiram identificar vários eventos hidrotermais presentes na área.

Veios Hidrotermais Esses eventos hidrotermais estão relacionados à formação de (1) veios pré-intrusão e presentes nos metamorfitos (VHM), (2) veios principais que se seguiram ao alojamento da intrusão com direção geral $\mathrm{N} 80^{\circ} \mathrm{W}$ e forte mergulho para SW (VHP) e (3) veios finais (VHF). Os primeiros são de origem metamórfica, sendo encontrados nas rochas dos grupos Babacu e Lagoa Seca e compostos de quartzo, topázio e sulfetos. Os VHP, de maior representatividade na jazida, estão relacionados ao episódio intrusivo do granito Musa e cortam a sua cupola greisenizada assim como as rochas do Supergrupo Andorinhas. Seu conteúdo mineral consiste essencialmente de quartzo, topázio, fluorita, mica branca, sulfetos e óxidos (principalmente wolframita). Já os VHF são tardios e secionam todos os tipos litológicos presentes na jazida. Possuem pouca representatividade espacial e são fundamentalmente constituídos de quartzo. 
Tabela 1 - Tipos de inclusões fluidas (IF) estudadas em rochas da jazida Pedra Preta (Rios 1995)

\begin{tabular}{|c|c|c|c|c|c|c|c|}
\hline $\begin{array}{c}\text { TIPOS } \\
\text { DE } \\
\text { IF }\end{array}$ & $\begin{array}{l}\text { TIPO 1 } \\
\mathrm{H}_{2} \mathrm{O}_{+} \\
\mathrm{CH}_{4}+ \\
\mathrm{CO}_{2}+ \\
\mathrm{NaCl}^{2} \\
\mathrm{CaCl}_{3} \\
\end{array}$ & $\begin{array}{c}\mathrm{TIPO2 \textrm {A }} \\
\mathrm{H}_{2} \mathrm{O}+\mathrm{NaCl} \\
+\mathrm{FeCl}_{2}\end{array}$ & $\begin{array}{c}\mathrm{TIPO} 2 \mathrm{~B} \\
\mathrm{H}_{2} \mathrm{O}+\mathrm{CO}_{2}+ \\
\mathrm{NaCl}+\mathrm{CH}_{1}+ \\
\mathrm{CaCl} l_{2}\end{array}$ & $\begin{array}{c}\text { TIPO3A } \\
\mathrm{H}_{2} \mathrm{O}+\mathrm{CO}_{2}+ \\
\mathrm{NoCl}+\mathrm{CaCl}_{2}\end{array}$ & $\begin{array}{c}\mathrm{TPO} 3 \mathrm{BH} \\
\mathrm{H}_{2} \mathrm{O}+ \\
\mathrm{NaCl}^{2} \\
\mathrm{CaCl}_{2}\end{array}$ & $\begin{array}{c}\text { F TIPO 4 } \\
\mathrm{H}_{2} \mathrm{O}+ \\
\mathrm{NaCl}^{+} \\
\mathrm{CaCl}_{2}+ \\
\mathrm{KCl}\end{array}$ & $\begin{array}{c}\text { IF TIPO5 } \\
\mathrm{H}_{2} \mathrm{O}+ \\
\mathrm{NaCl}\end{array}$ \\
\hline MINERAL & $\underset{1}{\text { QLARTZO }}$ & $\underset{3}{\text { OUARTOO }}$ & $\underset{1}{\text { QLAARTO }}$ & $\begin{array}{l}\text { TOPANDO } \\
\text { FLUORTIA }\end{array}$ & $\begin{array}{l}\text { TOPADO } \\
\text { FLUORTAA }\end{array}$ & OUAGTZO & 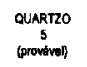 \\
\hline$\underset{\text { (velas) }}{\text { ROCHA }}$ & $\min$ & 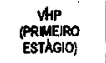 & $\begin{array}{c}\text { NPP } \\
\text { PRAMERO } \\
\text { ESTAGDO }\end{array}$ & 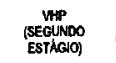 & 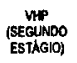 & VHe & MHF \\
\hline
\end{tabular}

A formação dos VHP envolveu dois estágios (Rios et al, submetido à publicação). No primeiro foi precipitado abundante quartzo, a partir inicialmente de fluidos salinos aquosos $\left(\mathrm{NaCl}-\mathrm{FeCl}_{2}-\mathrm{H}_{2} \mathrm{O}\right)$, derivados da cristalização do magma granítico, e, em seguida, da mistura desses fluidos com outros oriundos das rochas encaixantes e de composição aquo-carbônica $\left[\mathrm{NaCl}-\left(\mathrm{CaCl}_{2}\right)-\mathrm{CH}_{4}-\mathrm{H}_{2} \mathrm{O} \pm \mathrm{CO}_{2}\right]$. O segundo estágio está relacionado à reabertura do sistema de fraturas que havia anteriormente acolhido os filões de quartzo e é caracterizado pela entrada de fluidos enriquecidos em flúor que levaram à precipitacão de topázio e fluorita. $\mathrm{O} \mathrm{CO}_{2}$, de início abundante nesses fluidos, representados pelo sistema $\mathrm{NaCl}-\mathrm{CaQ} 2-\mathrm{CO}_{2}-\mathrm{H}_{2} \mathrm{O}$, exauriu-se completamente durante a deposição da fluorita. A wolframita foi, em geral, precipitada anteriormente ao topázio como bem demonstram as auréòlas deste ortossilicato circundando vários cristais daquele oxido. Da mesma forma, os VHF foram gerados em dois estágios, tendo no primeiro predominado fluidos aquosos salinos e quentes ( $\left.\mathrm{NaCl}-\mathrm{KCl}-\mathrm{CaCl}_{2}-\mathrm{H}_{2} \mathrm{O}\right)$ e no segundo fluidos de menor salinidade, temperatura mais baixa e composição mais simples $\left(\mathrm{NaCl}-\mathrm{H}_{2} \mathrm{O}\right)$.

Estudos detalhados de inclusões fluidas (Rios et al. 1997, Rios et ai. submetido à publicação) foram desenvolvidos em cristais de topázio, de fluorita e de quartzo de várias gerações, amostrados nos veios da jazida Pedra Preta (Tab. 1). Os dados indicaram condicões análogas para temperatura, fugacidade de oxigênio e pressão litostática nos setores inferior e superior da jazida. Entretanto, foram verificadas diferenças composicionais nos fluidos encontrados em uma mesma geração de quartzo dos VHP que foram amostrados em menores e maiores profundidades. No setor inferior, estudado entre 160 e $320 \mathrm{~m}$, os VHP cortam as zonas apicais do granito e somente os metarenitos do Grupo Lagoa Seca, enquanto no setor superior eles cortam as rochas de ambos aqueles grupos. E marcante o fato de os fluidos aquo-carbônicos mostrarem maiores teores de metano no setor superior comparativamente aos do setor inferior, o que foi interpretado como resultado de sua transformação para $\mathrm{CO}_{2}$ a maiores profundidades em resposta às condições francamente oxidantes impostas pelo alojamento do corpo Musa (Magalhães et al. 1994). Outras diferenças existem como bem demonstraram as análises isotópicas de oxigênio realizadas em amostras de quartzo, micas e wolframita, as quais também serviram para a obtenção de dados termométricos independentes daqueles conseguidos com inclusões fluidas.

ESTUDO DE ISÓTOPOS DE OXIGÊNIO Com base na composição isotópica do fluido aquoso relacionado com a deposição do minério ou com a alteração das rochas encaixantes, pode-se inferir a origem dos fluidos e a temperatura de formação dos minerais que dele foram precipitados (Faure 1977).

A inexistência no Brasil de laboratórios em condições de analisar isótopos de hidrogênio em minerais restringiu o presente estudo à composição isotópica do oxigênio de quartzo, micas e wolframita da jazida de Pedra Preta e, indiretamente, também às composições isotópicas dos fluidos que os precipitaram.

As frações de quartzo estudadas pertencem preponderantemente aos VHP. A wolframita está cortada por vênulas microscópicas de scheelita (perfazendo de 5 a $10 \%$ do volume), o que tornou impraticável a separação de frações puras de wolframita. As análises com microssonda da mica branca revelaram tratar-se de fengita (variedade de muscovita com razão $\mathrm{Si} / \mathrm{Al}>3$ na posição tetraédrica e significativa substituição do $\mathrm{Al}$ octaédrico por $\mathrm{Fe}$ e $\mathrm{Mg}$ ) com composição média $\left(\mathrm{Ki}_{1,90} \mathrm{Na}_{0,24} \mathrm{Ca}_{0,03}\right)\left(\mathrm{Fe}_{0,88} \mathrm{Mg}_{0.22} \mathrm{Mn}_{0,02} \mathrm{Ti}_{0,03} \mathrm{Al}_{4,18}\right)$

$\left[\mathrm{Si}_{6,94} \mathrm{Al}_{1,06} \mathrm{O}_{22}\right](\mathrm{OH})_{2}$ para 24 átomos de oxigênio.

Comparação de $\delta^{18} \mathrm{O}$ no Granito Musa e nos veios hidrotermais Os dados isotópicos de oxigênio obtidos em quartzo, wolframita e mica branca (Fig. l) são representados pela variável ô (desvio per mil, $\%$ ) que é calculada pela equação (1)

$$
\delta^{18} \mathrm{O}=\left[\left({ }^{18} \mathrm{O} /{ }^{16} \mathrm{O}\right)_{\mathrm{a}} /\left({ }^{18} \mathrm{O} /{ }^{16} \mathrm{O}\right) \text { smow - } 1\right] \mathrm{X} 10^{3}
$$

onde o subscrito "a" refere-se à amostra de interesse e SMOW (Standard Mean Ocean Water) é o padrão internacionalmente adotado (Faure 1977).

05 FRACIONAMENTOS DE PARES MINERAIS Os dados sobre a composição isotópica de oxigênio do quartzo mostram variação com a profundidade na área da jazida Pedra Preta (Fig.2A). Os valores de $\mathrm{S}^{18} \mathrm{O}_{\text {cuartzo }}$ do granito e dos VHP do setor inferior da jazida são similares e menores do que os do VHP do setor superior. Enquanto as amostras de quartzo dos VHP do setor inferior ficaram livres de contaminação, pode ter havido alguma mistura do quartzo dos VHF com aquele dos VHP do setor superior, porém há a certeza de que o material proveniente dos VHP foi, de longe, preponderante.

As diferenças entre os valores de $\delta{ }^{18} \mathrm{O}_{\text {ouartzo }}$ observadas nos dois setores, de até $2,51 \%$, não podem ser atribuídas à temperatura, pois em ambos prevaleceu, de acordo com os dados microtermométricos, essencialmente a mesma faixa termal de 230 a $350^{\circ} \mathrm{C}$, se bem que no setor superior tenham sido registrados alguns valores abaixo e acima desses limites (Rios 1995). Aquelas diferenças devem refletir, por outro lado, as composições isotópicas de oxigênio das variedades litológicas dominantes no setor inferior (rochas graníticas e metarenitos do Grupo Lagoa Seca) e no setor superior (rochas metabásicas do Grupo Babaçu e metarenitos do Grupo Lagoa Seca) com as quais os fluidos hidrotermais interagiram. Devem refletir, sobretudo, diferenças composicionais dos fluidos aquo-carbônicos que, no setor superior, foram bem mais enriquecidos em $\mathrm{CH}_{4}$.

São escassos os estudos sobre isótopos de oxigênio em wolframita de depósitos minerais existentes na literatura (Landis \& Rye 1974, Kelly \& Rye 1979, Campbell et al. 1984, Higgins \& Kerrich 1982, Só 6 Yun 1994). Registram-se valores entre 0,6 e 5,8\% com grande variação mesmo em um único depósito que, segundo aqueles autores, pode ser devida a vários processos, desde a contaminação com scheelita até a mistura com águas meteóricas ou ainda a separação de uma fase rica em $\mathrm{CO}_{2}$ a partir de um fluido homogêneo. Um valor isolado para a wolframita de Pedra Preta $\left(\delta^{18} \mathrm{O}=0,85 \%\right)$ desaconselha grandes ilações. E sabido, contudo, que essa wolframita contém certa quantidade de scheelita e que a entrada de água meteórica no sistema Pedra Preta somente se deu durante o $2^{\circ}$ estágio de formação dos VHF. Ademais, é improvável que fluidos altamente pressurizados, como aqueles responsáveis pelo fraturamento hidráulico que levou à formacão dos VHP (Rios 1995), permitissem a incursão de água meteórica, cujas pressões em processos de resfriamento de corpos plutônicos, por convecção, não excedem 100bar/km (Cathles 1977). Quanto à imiscibilidade do $\mathrm{CO}_{2}$, constatou-se, em Pedra Preta, apenas ocorrência local desse fenômeno.

Há também apenas um dado isotópico para a mica branca de Pedra Preta $\left(\delta{ }^{18} \mathrm{O}=5,44 \%\right)$ e refere-se a uma amostra dos VHP coletada a $255 \mathrm{~m}$ de profundidade. Como já foi visto, trata-se de fengita e apesar de ela desviar-se significativamente da composição estequiométrica da muscovita, os dados isotópicos desta última foram, não obstante, usados nas equações de fracionamento.

OS FRACIONAMENTOS DE MINERAIS-ÁGUA O valor de $\delta^{18} \mathrm{O}$ para o quartzo da amostra MU-25, coletada em afloramento do granito Musa a cerca de $2 \mathrm{~km}$ da jazida, presumivelmente fora da área de influência do sistema hidrotermal Pedra Preta, foi medido em 7,61\%. Trabalhos experimentais sobre o granito Jamon acusaram um intervalo de cristalização do magma entre cerca de 850 e $700^{\circ} \mathrm{C}$ com grande parte do quartzo formando-se próximo do ponto eutético e teores de água constantes a levemente crescentes (Scaillet et al. 1995). Dado o fato de esse granito pertencer à mesma suíte anorogênica e ser petrologicamente muito semelhante à intrusão Musa, admitiu-se também uma história termal análoga. Para uma temperatura de $750^{\circ} \mathrm{C}$, calculou-se, com base na equação de fracionamento quartzo-água de Clayton et al. $(1972), \delta{ }^{18} \mathrm{O}=6,93 \%$ para os fluidos aquosos em equilíbrio com 0 quartzo primário, que é um valor inteiramente compatível com a origem magmática desses fluidos (Taylor 1979).

Também foram calculadas as composições isotópicas de oxigênio dos fluidos hidrotermais coexistindo com quartzo e wolframita dos VHP (Fig. 2B) a partir das equacões de fracionamento quartzo-água de Clayton et al. (1972) e de wolframita-água de Zhang et al. (1994), no intervalo de 240 a $300^{\circ} \mathrm{C}$, o qual, de acordo como os dados obtidos com inclusões fluidas em quartzo (Rios 1995), é o mais freqüente em ambos os setores da jazida. Pode-se observar que todos os valores de $\delta^{18} \mathrm{O}_{\text {flúido }}$ são inferiores a $2 \%$, bem abaixo portanto daqueles atribuídos 


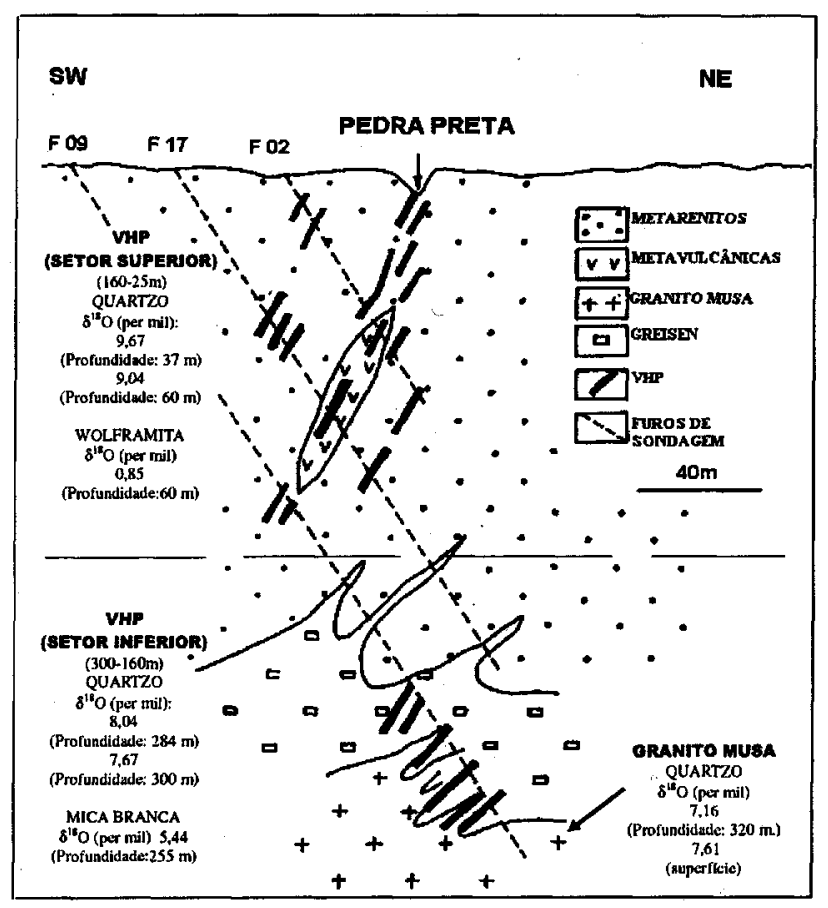

Figurall - Seção SW-Ms da jazida Pedra Preta (modificada de Santos 1987) na qual são fornecidos os resultados isotópicos de oxigênio referentes ao quartzo do granito Musa e ao quartzo, wolframita e mica branca dos veios hidrotermais principais (VHP)

às águas magmáticas (Taylor 1979). Também observam-se valores mais baixos de $\delta^{18} \mathrm{O}_{\text {fluido }}$ no setor inferior, o que era de se esperar tendo em vista os menores valores de $\delta{ }^{18} \mathrm{O}_{\text {quartzo }}$ nesse setor. Esses dados sugerem a forte interferência de fluidos de outra natureza, tais como os de composição aquo-carbônica, empobrecidos em ${ }^{18} \mathrm{O}$ e de caráter redutor (presença de $\mathrm{CH}_{4}$ ). Poderiam sugerir também reequilíbrio das águas magmáticas com o granito a temperaturas mais baixas (Higgins 1995 b), se bem que nesse caso os valores de $\delta{ }^{18} \mathrm{O}_{\text {nuido }}$ não deveriam ser inferiores a 3-4\%o (Taylor 1968).

Apesar da constatação de alguma imiscibilidade dos fluidos aquocarbônicos no sistema Pedra Preta (Rios 1995), a perda de $\mathrm{CO}_{2}$ por esse processo não parece ter sido importante quando da precipitação da wolframita. Dados espectroscópicos de infra-vermelho na wolframita e microtermométricos e de espectrometria RAMAN no topázio revelaram que ambos minerais foram precipitados de fluidos ricos em $\mathrm{CO}_{2}$. Assim, na formação dos VHP, não se pode explicar os baixos valores de $\delta{ }^{18} \mathrm{O}_{\text {nuido }}$ pela separação do $\mathrm{CO}_{2}$ que, quando ocorre, deixa os fluidos aquosos residuais empobrecidos em ${ }^{18} \mathrm{O}$ (Higgins \& Kerrich 1984). Em outras palavras, os valores de $\delta{ }^{18} \mathrm{O}$ encontrados para o quartzo e fluidos dos VHP devem refletir fundamentalmente a composição isotópica do oxigênio de fluidos não-magmáticos, com prolongada residência nas rochas regionais, que foram acionados em decorrência da abertura de fraturas relacionadas com o alojamento do granito Musa.

É mais difícil explicar os maiores valores de $\delta{ }^{18} \mathrm{O}$ encontrados no setor superior da jazida. As evidências microtermométricas mostram que a deposição do quartzo no setor inferior se deu preferencialmente durante $\mathrm{o} 1^{\circ}$ estágio de formação dos VHP, sendo modificados isotopicamente quando da entrada dos fluidos aquo-carbônicos do $2^{\circ}$ estágio. $\mathrm{O}$ ambiente relativamente mais oxidante do setor inferior e que levou à quase completa transformação do $\mathrm{CH}_{4} \mathrm{em} \mathrm{CO}_{2}$, certamente favoreceu o enriquecimento de $\mathrm{CO}_{2}$ nos fluidos que ascenderam antes que pudessem se reequilibrar com o quartzo. No setor superior, por outro lado, grande parte do quartzo analisado é contemporânea com a wolframita, mineral que foi precipitado de fluidos enriquecidos em $\mathrm{CO}_{2}$, como já mencionado. A partição de ${ }^{18} \mathrm{O}$ é favorecida em fluidos contendo $\mathrm{CO}_{2}$ relativamente àqueles em que esse gás está ausente (Higgins 1985a e 1985b, Só \& Yun 1994, dentre outros), de modo que era de esperar, em tendo havido equilíbrio, maiores valores de $\delta^{18} \mathrm{O}$ tanto no quartzo como nos fluidos que o precipitaram.

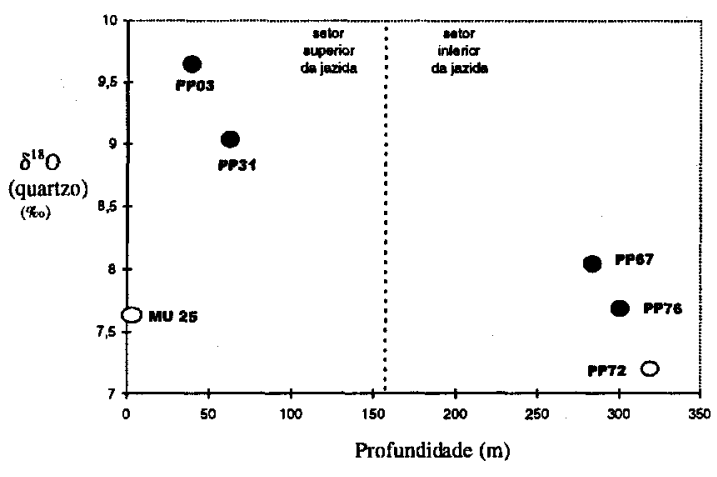

A

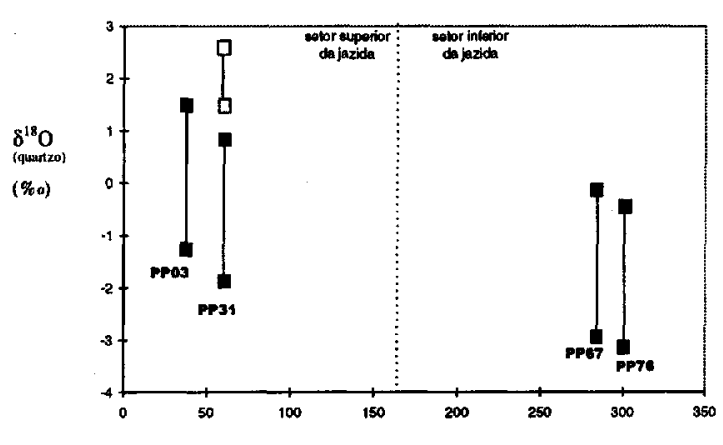

$\mathbf{B}$

Profundidade $(\mathrm{m})$

Figura 2 - A. Composição isotópica de oxigênio em quartzo dos VHP

(๑) e do granito ( $O$ ), mostrando os maiores valores de $\delta^{18} O$ no setor superior da jazida. A linha tracejada marca, a aproximadamente $160 \mathrm{~m}$ da atual superficie de erosão, a separação entre os setores superior e inferior. A amostra MU25 foi coletada em afloramento do granito a cerca de $2 \mathrm{~km}$ da jazida. B. Composições isotópicas de oxigênio dos fluidos hidrotermais presentes nos VHP coexistindo como quartzo ( $)$ e wolframita (प), calculadas a partir das equações de fracionamento quartzo-água de Clayton et al. (1972) e wolframita-água de Zhang et al. (1994) na faixa de temperatura entre 240 e $300^{\circ} \mathrm{C}$ (ver texto).

Cálculos feitos com base na equação de fracionamento wolframitaágua (Zhang et al. 1994) acusaram valores de $\delta{ }^{18} \mathrm{O}_{\text {fluido }}$, para a faixa de temperatura $240-300^{\circ} \mathrm{C}$, entre 1,59 e $2,64 \%$ o, um pouco acima daqueles obtidos para os fluidos em equilíbrio com quartzo dos VHP do setor superior (Fig. 2B) no mesmo intervalo termal. $\mathrm{O} \delta{ }^{18} \mathrm{O}$ de equilíbrio entre quartzo e wolframita a $300^{\circ} \mathrm{C}$, calculado pela equação de fracionamento de Zhang et al. (1994), é de $9,30 \%$, que é $1,1 \%$ maior do que aquele obtido pelos resultados analíticos, os quais forneceram valores de $\delta{ }^{18} \mathrm{O}$ iguais a $9,00 \%$ e $0,85 \%$ respectivamente para quartzo e wolframita de uma mesma amostra coletada a $60 \mathrm{~m}$ de profundidade. Essa pequena diferença pode indicar uma temperatura de equilíbrio mais alta que $300^{\circ} \mathrm{C}$, o que tem suporte nos dados microtermométricos (temperaturas determinadas sem correção de pressão, portanto mínimas) e texturais observados para quartzo, wolframita e topázio.

Geotermometria Para determinar-se, por meios isotópicos, a temperatura de dois minerais é necessário conhecer-se o coeficiente de fracionamento entre eles, o qual se obtém calculando-se, inicialmente, os fracionamentos entre o mineral e a água. A seguir, calibra-se o fracionamento entre o par mineral $\left(\alpha_{\mathrm{M}-\mathrm{N}}\right)$, variável esta que é relacionada à temperatura pela equação $(2)$

$$
1000 \text { In } \alpha \mathrm{M}-\mathrm{N}=\mathrm{A}\left(10^{6} / \mathrm{T}^{2}\right)+\mathrm{B}
$$

onde A e B são constantes e T é a temperatura absoluta (Faure 1977, Sial \& McReath 1984).

Os minerais selecionados para estudos de termometria isotópica em Pedra Preta foram o quartzo, a mica branca e a wolframita. A curva de fracionamento wolframita- $\mathrm{H}_{2} \mathrm{O}$ e o geotermômetro do par quartzowolframita foram, até agora, pouco estudados. De qualquer modo, o fracionamento isotópico entre quartzo e wolframita é grande, constituindo um geotermômetro mais sensível do que o quartzo-muscovita (Shieh\&Zhang1991). 
No depósito de Pedra Preta, a wolframita precipitou quando da oxidação dos fluidos aquo-carbônicos que haviam entrado anteriormente no sistema. O quartzo e a wolframita utilizados no par mineral foram aqueles para os quais as evidências de contemporaneidade eram marcantes, a exemplo da textura de intercrescimento que eles apresentam. Como esses dois minerais foram precipitados de um mesmo fluido rico em $\mathrm{CO}_{2}$ e as equações de fracionamento quartzo-água e wolframita-água forneceram valores de $\delta^{18} \mathrm{O}_{\text {fluído }}$ equiparáveis, admitiu-se que ambos foram depositados em equilíbrio isotópico.

Os valores de 1000 In a, da eurva do par quartzo-wolframita, foram referenciados à curva de Shieh \& Zhang (1991), obtendose a temperatura de $321{ }^{\circ} \mathrm{C}$ para $\delta^{18} \mathrm{O}_{\text {quartzo }}=9,04 \%$ e para $\delta^{18} \mathrm{O}_{\text {wolf }}$ $=0,85 \%$ o. De outra feita, aplicando-se a equação (3)

$\delta$ quartzo $-\delta$ wolf $=-1,04 \times 10^{6} / \mathrm{T}^{2}+10,74 \times 10^{3} / \mathrm{T}-6,27$

(Zhang et al 1994), calculou-se $\mathrm{T}=628^{\circ} \mathrm{K}\left(356^{\circ} \mathrm{C}\right)$, temperatura esta que parece mais próxima da verdadeira temperatura de precipitação do minério. Devido à contaminação de scheelita, esses valores certamente incorporam algum erro, cuja extensão não pôde ser avaliada, mas acredita-se não levar a diferenças significativas.

Seguiu-se o mesmo procedimento para o par isotópico quartzomuscovita. Com base nas equações de fracionamento do par quartzomuscovita de Clayton et al (1 972), Bottinga \& Javoy (1 973) e Blattner (1975) foram calculadas, para $\delta{ }^{18} \mathrm{O}_{\text {musc }}=5,44 \%$ e $\delta^{18} \mathrm{O}_{\text {quatzo }}=7,86 \%$ (valor médio do setor inferior), temperaturas entre 580 e $756^{\circ} \mathrm{C}$, que são demasiadamente elevadas para o sistema hidrotermal de Pedra Preta e devem refletir a incerteza com os dados de calibração. Adotouse, então, a curva proposta por Shieh \& Zhang (1991) que foi calibrada de modo a abranger temperaturas do ambiente hidrotermal. Ainda assim, os resultados obtidos ficaram próximos de $410^{\circ} \mathrm{C}$ que é superior ao valor determinado para a precipitação da wolframita. Como a mica foi um dos últimos minerais a se cristalizar nos VHP, portanto posterior à wolframita e ao topázio, a temperatura de $400^{\circ} \mathrm{C}$ deve ser vista com reservas. Além do baixo fracionamento isotópico quartzo-muscovita, algum erro pode advir do uso do coeficiente de fracionamento da muscovita quando, na realidade, a mica branca de Pedra Preta é a variedade fengita.

CONCLUSÕES Os estudos isotópicos de oxigênio na jazida Pedra Preta sugerem a participação de fluidos hidrotermais empobrecidos em ${ }^{18} \mathrm{O}$ na formação dos veios VHP. De origem externa ao granito Musa, esses fluidos, com composição aquo-carbônica $\left(\mathrm{H}_{2} \mathrm{O}+\mathrm{CH}_{4}+\right.$ sais \pm $\mathrm{CO}_{2}$ ), foram oxidados nas zonas apicais do granito, onde o $\mathrm{CH}_{4}$ foi quase que totalmente transformado em $\mathrm{CO}_{2}$. Ao ascenderem, já enriquecidos em $\mathrm{CO}_{2}$, tornaram-se também enriquecidos em ${ }^{18} \mathrm{O}$ propiciando a precipitação de quartzo com maiores $\delta^{18} \mathrm{O}$ que no setor inferior. A aplicação do geotermômetro isotópico quartzo-wolframita forneceu temperaturas de $356^{\circ} \mathrm{C}$ (Zhang et al 1994) que são compatíveis com os dados microtermométricos (Rios 1995). A contaminação da wolframita por scheelita pode ter falseado esses valores, mas acreditase que o erro foi de pequena monta. De qualquer modo, eles devem ser tomados como temperaturas mínimas de precipitação do minério. Já o par quartzo-muscovita (Shieh \& Zhang 1991) acusou temperaturas da ordem de $410^{\circ} \mathrm{C}$ que devem também ser interpretadas com cautela, tendo em vista as relações paragenéticas serem indicativas da posteridade da mica branca relativamente à wolframita ou mesmo o uso do coeficiente de fracionamento da muscovita quando, na realidade, a mica branca de Pedra Preta tem composição fengítica.

Agradecimentos À CAPES pela bolsa de doutorado a FJR; ao CG-UFPA que acolheu FJR em seu programa de doutorado; à professora Valderez Ferreira (LABISE-UFPE) pela colaboração nas linhas de extração para estudos isotópicos; ao CDTN-CNEN e CPMTCUFMG pelo apoio de infra-estrutura; à Docegeo do Distrito da Amazônia pelo apoio logístico no campo e fornecimento de amostras de testemunhos de sondagem da jazida Pedra Preta; e a dois relatores anônimos da RBG que, com suas críticas e sugestões, muito enriqueceram o conteúdo deste trabalho.

\section{Referências}

Blattner, P. 1975. Oxygen isotopic composition of fissure-grown quartz, adularia, and calcite from Broadlands geothermal field, New Zealand with an appendix on quartz-K-feldspar-calcite-muscovite oxygen isotope geothermometer. Amer. J. Sci. 275:785-800

Bottinga, Y \& Javoy, M. 1973. Comments on oxygen isotope geothermometry. Earth Plan. Sci. Letters 20:250-265

Campbell, A.R.; Rye, D.; Petersen, U. 1984. An hydrogen and oxygen isotope study of the San Cristobal Mine, Peru: implications of the role of water to rock ratio for the genesis of wolframite deposits. Earn. Geol., 79:1818-1832.

Cathles, L.M. 1977. An analysis of the cooling intrusives by ground-water convection which includes boiling. Earn. Geol. 72:804-826

Clayton, R.N.; O'Neil, J.R.; Mayeda, T.K. 1972. Oxygen isotope exchange between quartz and water. J. Geoph. Res. 77:3057-3067

Cordeiro, A.A.C.; Alves, C.A.; Fonseca, L.R.; de Nascimento, J.A.S.; Azevedo, L.L.; Santos, F.F. 1984. Pesquisa geológica da jazida de wolframita de Pedra Preta, Rio Maria. In: Simpósio de Geologia da Amazônia, 2, Manaus, 1984. Anais... Manaus. MME-DNPM. p. 374-390.

Cordeiro, A.A.C.; Alves, C.A.; Biagini, D.O.; Fonseca, L.R.; de Nascimento, J.A.S.; Azevedo, L.L.; Santos, F.F. 1988 Depósito de wolframita de Pedra Preta, sul do Pará. In: Congresso Brasileiro de Geologia, 35, Belém, 1988. Província Mineral de Carajás - Litoestratigrafia e principais depósitos minerais. Anexo aos anais...Belém, CVRD/SBG.p.161-165.

Dall'Agnol, R.; Lafon, J. M.; Macambira, M: J. B. 1994. Proterozoic anorogenic magmatism in the Central Amazonian Province, Amazonian Craton: geochronological, petrological and geochemical aspects. Mineral. Petrol. 50:113-138

DOCEGEO. 1988. Revisão litoestratigráfica da Província Mineral de Carajás. In: Congresso Brasileiro Geologia 35, Belém, 1988. Província Mineral de Carajás -Litoestratigrafia e principais depósitos minerais. Anexo aos anais... Belém, CVRD/SBG, p. 11-54.

Faure, G. 1977. Principles of isotope geology. New York, John Wiley \& Sons. 589p.

Gastai, M.C. 1987. Maciço granítico Musa: mapeamento, petrologia e petroquímica. Belém, 307p. (Tese de mestrado, CG/UFPA)

Higgins, N.C. 1985a. Wolframite deposition in a hydrothermal vein system: the Grey River tungsten prospect, Newfoundland, Canada. Econ. Geol. 80:1297-1327.

Higgins, N.C. 1985b. Moderately depleted oxygen isotope composition of waters associated with tin- and tungsten-bearing quartz veins: an evaluation of isotopic models. In: Herbert, H.K. \& So, S.E. (ed) Stable isotopes and fluid processes in mineralization. Publ. 23, Univ. Western Australia, p. 204-214

Higgins, N.C. \& Kerrich, R. 1984. Progressive ${ }^{18} \mathrm{O}$ depletion during CÜ2 separation from carbon dioxide-rich hydrothermal fluid: evidence from the Grey River tungsten deposit, Newfoundland. Can J. Earth Sci. 19:2247-2257

Kelly, W.C. \& Rye, R.0.1979. Geologic, fluid inclusion, and stable isotope studies of the tin-tungsten deposits of Panasqueira, Portugal. Econ. Geol. 74:1721-1822

Landis, G.P. \& Rye, R.0.1974. Geologic, fluid inclusion and stable isotope studies of the Pasto Bueno tungsten-base metal ore deposit, northern Peru. Econ. Geol. 69:1025-1059

Macambira, M.J.B. \& Lancelot, J. 1992. Idade U-Pb em zircões de metavulcânicas do "greenstone" do Supergrupo Andorinhas, deliminante da estratigrafia arqueana de
Carajás, Estado do Pará In: Congresso Brasileiro de Geologia, 37, São Paulo, 1992. Resumos Expandidos... São Paulo, SBG. v. 2, p. 188-189

Machado, N.; Lindenmayer, Z.; Krogh, T.E.; Lindenmayer, D. 1991. U-Pb geochronology of Archean magmatism and basement reactivation in Carajás area, Amazon Shield, Brazil. Precambrian Res. 49:327-354.

Magalhães, M.S.; Dall'Agnol, R.; Sauck, A.W.; Luiz, J.G. 1994. Susceptibilidade magnética: um indicador da evolução petrológica de granitóides da Amazônia. Revista Brasileira de Geociências 24:139-149

Pimentel, M.M. \& Machado, N. 1994. Geocronologia U-Pb dos terrenos granito-greenstone de Rio Maria, Pará. In: Congresso Brasileiro de Geologia, 38, Camboriu, 1994. Resumos Expandidos... Camboriu, SBG. v. 2, p. 390-391.

Rios, F.J. 1995. A jazida de wolframita de Pedra Preta, granito Musa (PA): estudo dos fluidos mineralizantes e isótopos estáveis de oxigênio em veios hidrotermais. Belém, 214p. (Tese de doutorado, CG-UFPA).

Rios, F.J.; Fuzikawa, K. Villas, R.N.; Pimenta, M.A. 1997. Estudo de inclusões fluidas modificadas em quartzo de veios hidrotermais com wolframita, jazida de Pedra Preta, Amazônia Oriental (PA). Rev. Escola Minas, 50:40-44

Rios, F. J.; Villas, R.N.; Fuzikawa, K. Fluid and metallogenetic evolution of the Pedra Preta wolframite ore deposit, East Amazon, Brazil (submetido a Mineralium Deposita).

Santos, J.F.D. 1987. Tipologia e gênese do depósito de tungstênio de Pedra Preta (PA). São Paulo, 93p. (Tese de mestrado, IG-USP)

Scaillet, B; Pichavant, M; Dall'Agnol, R. 1995. Experimental determination of phase equilibria of the Jamon granite, Eastern Amazonian Province. In: Symposium on Rapakivi Granites and Related Rocks, Belém, 1995. Abstracts... Belém, IGCP, Project 315. p. 69

Shieh, Y. \& Zhang, G.X. 1991. Stable isotope studies of quartz-vein type tungsten deposits in Dajishan mine, Jiangzi province, Southeast China. In: Taylor, H.P.; O'Neil, J.R ; Kaplan, I. ed. Stable isotope geochemistry: a tribute do Samuel Epstein. The Geochemical Society, Spec. Publ. 3, p. 425-435.

Sial, A.N. \& McReath, I. 1984. Petrologia ignea: os fundamentos e as ferramentas. SBG-CNPq-Bureau Ltda. 179p.

So, C.S. \& Yun, S.T. 1994. Origin and evolution of W-Mo-producing fluids in a granitic hydrothermal system: geochemical studies of quartz vein deposit around Susan granite, Hwanggangri district, Republic of Korea. Econ. Geol., 89:246-267.

Taylor, H.P. 1968. The oxygen isotope geochemistry of igneous rocks. Cont. Mineral Petrol. 19:1-71

Taylor, H.P. 1979. Oxygen and hidrogen isotope relationships in hydrothermal ore deposits. In: Barnes, H.L. ed. Geochemistry of hvdrothermal ore deposits. New York, John Wiley \& Sons, p. 236-277

Zhang, L.G.; Lui, X.L.; Chen, Z.S.; Zhou, H.B. 1994. Experimental investigations of the oxygen isotope fractionations in cassiterite and wolframite. Econ. Geol., $89: 150-157$.

Manuscrito A-936 Recebido em 20 de setembro de 1997 Revisão dos autores em 10 de março de 1998 Revisão aceita em 15 de março de 1998 